\title{
Aprendizaje turístico por descubrimiento significativo mediante viajes académicos de estudio: estrategia y práctica de sustentabilidad. Puerto Peñasco, México
}

Tourist Learning by Significant Discovering through Academic Study Trips: strategy and practice of sustainability. Puerto Peñasco, México

Tourist descoberta significativa a aprendizagem através de viagens de estudo académicas: estratégia e prática de sustentabilidade. Puerto Penasco, México

\section{Manuel Ramón González Herrera}

Universidad Autónoma de Ciudad Juárez, México manglez04@yahoo.es

Edione Teixeira de Carvalho Instituto Federal de Mato Grosso, Brasil edione.carvalho@svc.ifmt.edu.br

\section{Resumen}

El desarrollo emprendedor es un factor clave de éxito para el turismo sustentable, el cual requiere de una formación práctica innovadora, adecuada a las tendencias del mercado turístico. El objetivo de la investigación fue desarrollar una experiencia de aprendizaje turístico por descubrimiento significativo mediante los viajes académicos de estudio en el destino turístico litoral Puerto Peñasco, a través de la materia Planificación Turística del Programa de Turismo de la Universidad Autónoma de Ciudad Juárez (UACJ). Se implementó una metodología experimental, en la que se utilizaron métodos empíricos, teóricos y estadístico-matemáticos. La actividad práctica aplicó el enfoque geoespacial al estudio de un destino mediante la interpretación del proceso de producción del espacio turístico, así como el reconocimiento del modelo de implantación, el inventario y evaluación de recursos turísticos y el análisis del esquema de estructuración del espacio turístico. Los resultados permitieron comprender y 
justificar la contribución del proceso de aprendizaje práctico a la formación de conocimientos turísticos incorporando los viajes académicos de estudio extracurriculares al desarrollo del aprendizaje turístico por descubrimiento significativo como estrategia y práctica de sustentabilidad.

Palabras clave: conocimiento, turístico, aprendizaje, viajes académicos de estudio, sustentable.

\section{Abstract}

Entrepreneurial development is a key success factor for sustainable tourism, which requires practical innovative training, adequate to the tourism market trends. The objective of the research was develop an experience of tourist learning by Significant Discovering through academic study trips to the tourist coastal destination Puerto Peñasco, through the subject Planning Tourism of the Tourism Program of the Autonomous University of Ciudad Juárez (UACJ by its name in Spanish). We implemented an experimental methodology, which used methods empirical, theoretical and statistical - mathematical. The activity practice applied the geospatial approach to the study of a destination through the interpretation of the production process of the tourist space, as well as the recognition of model implementation, inventory and evaluation of tourist resources and analysis of the structuring of the space tourism scheme. The results allowed to understand and justify the contribution of the process of learning to the formation of tourist expertise incorporating academic extracurricular study trips to the development of the tourist learning by Significant Discovering as strategy and practice of sustainability.

Key words: knowledge, tourism, learning, academic study trips, Academic Travel, Travel Study, sustainable.

\section{Resumo}

O desenvolvimento empresarial é um fator chave de sucesso para o turismo sustentável, o que requer um inovador adequada formação prática para as tendências do mercado turístico. $\mathrm{O}$ objetivo da pesquisa era desenvolver uma experiência de turismo aprendizagem descoberta significativa de viagens de acadêmicos no destino turístico costa Puerto Peñasco, através da matéria Turismo Planejamento Turismo Programa da Universidade Autônoma de Ciudad Juarez (UACJ) - uma metodologia experimental, no qual foram utilizados métodos empíricos, teóricos e estatísticos e matemáticos foi implementado. atividade prática aplicada abordagem geoespacial 
para o estudo de um destino interpretando o processo de produção do espaço turístico e reconhecimento de padrões de implementação, inventário e avaliação dos recursos turísticos e estruturação esquema de análise do espaço turístico. Os resultados permitiram-nos a compreender e justificar a contribuição do processo de aprender a formação incorporar o conhecimento de viagens turísticas desenvolvimento acadêmico extracurricular do turismo como estratégia de aprendizagem descoberta significativa e prática do estudo de sustentabilidade.

Palavras-chave: conhecimento, de turismo, de aprendizagem, estudo acadêmico, viagem sustentável.

Fecha recepción: Febrero 2016

Fecha aceptación: Julio 2016

\section{Introducción}

El desarrollo emprendedor es un factor clave de éxito para el turismo sustentable, ya que los emprendedores son el cimiento principal que permitirá el crecimiento sostenido del sector, cuya formación práctica e innovadora requiere del fomento de capacidades adecuadas a las nuevas tendencias que se registran en el mercado turístico. Por tal razón, es esencial entender esta dinámica desde la perspectiva formativa de los recursos humanos que en la actualidad demanda el turismo, cuya experiencia vivencial, práctica y participativa se ha convertido en un requisito obligatorio para los modelos educativos y planes de estudio de las diferentes carreras universitarias.

La premisa básica en que se sustenta la investigación se sostiene en el supuesto de que la profesionalización del turismo a través del aprendizaje significativo por descubrimiento, constituye un aspecto clave para las estrategias de desarrollo turístico sustentable. Esto es debido a que los egresados que han realizado viajes académicos de estudio durante su proceso formativo egresan con un mayor nivel de competencia laboral.

Relativo a la misma se elaboran los siguientes enunciados interrogativos con el fin de encontrar posibles respuestas y someterlas al debate de los especialistas e interesados en el tema: 
1) ¿Cuál es la contribución del proceso de aprendizaje práctico a la formación de nuevos conocimientos turísticos experienciales que responden a las necesidades del sector turístico?

2) ¿Qué valor se puede conceder al aprendizaje turístico por descubrimiento significativo guiado mediante los viajes académicos de estudio?

3) ¿Cuál puede ser la utilidad de los viajes académicos de estudio extracurriculares?

4) ¿Cómo operacionalizar e implementar los viajes académicos de estudio extracurriculares para favorecer el aprendizaje turístico por descubrimiento significativo y garantizar la eficiencia formativa de las actividades realizadas?

Por tanto, el objetivo general de la investigación es desarrollar una experiencia de aprendizaje turístico por descubrimiento significativo mediante los viajes académicos de estudio en el destino turístico litoral Puerto Peñasco, Sonora, a través de la materia Planificación Turística del Programa de Licenciatura en Turismo de la Universidad Autónoma de Ciudad Juárez (UACJ), como estrategia y práctica de sustentabilidad.

Para el logro del objetivo general se desarrollaron las siguientes etapas de investigación:

1) Análisis del componente práctico formativo del Programa de Licenciatura en Turismo de la UACJ, atendiendo a los viajes académicos de estudio en las modalidades de visitas y recorridos curriculares.

2) Propuesta de un instrumento para el desarrollo de viajes académicos de estudio extracurriculares.

3) Identificación de los conocimientos turísticos curriculares que sirven de soporte teórico al desarrollo de viajes académicos de estudio en la materia de Planificación Turística.

4) Implementación práctica y desarrollo de experiencias de aprendizaje turístico por descubrimiento significativo mediante un viaje académico de estudio extracurricular al destino litoral Puerto Peñasco, Sonora, orientado a la comprensión práctica de la sustentabilidad.

Los resultados obtenidos permitieron comprender y justificar la contribución del proceso de aprendizaje práctico a la formación de nuevos conocimientos turísticos experienciales, de forma tal que se responda a las necesidades prácticas del sector turístico incorporando los viajes académicos de estudio extracurriculares para el desarrollo del aprendizaje turístico por 
descubrimiento significativo con enfoque de sustentabilidad. Con base en los antecedentes teóricos elaborados y los supuestos metodológicos asumidos se presentan los resultados de la experiencia práctica desarrollada con los estudiantes de la Licenciatura en Turismo de la UACJ en el destino turístico litoral Puerto Peñasco, Sonora.

\section{Revisión de la literatura}

Bases teóricas para el aprendizaje turístico por descubrimiento significativo

De acuerdo con la sistematización teórica desarrollada por Traverso et al., con respecto al aprendizaje a través de la experiencia (2010), se señala que:

El aprendizaje es un proceso activo de interacción entre el individuo y el ambiente, que puede acontecer a lo largo de toda la vida y en todos los momentos de la experiencia humana. Por su parte, el conocimiento es el resultado de las transacciones entre esas experiencias subjetivas y objetivas; por lo tanto, el aprendizaje es el proceso de creación del conocimiento. El aprendizaje es concebido como un proceso donde las ideas son formadas y reformuladas a través de la experiencia, convirtiéndolo en un continuo reaprendizaje que se apoya en la experiencia de los individuos (Traverso et al., 2010, p. 367).

Consecuentemente, el aprendizaje turístico puede ser comprendido como el proceso activo de adquisición de conocimientos, habilidades, valores, capacidades y actitudes relativos al turismo, lo cual supone un cambio de comportamiento a favor del desarrollo turístico sustentable. El conocimiento turístico muchas veces es definido como tácito, pero la experiencia adquirida a través de la enseñanza formal es relevante para el desarrollo de las propias experiencias de los gestores turísticos (Traverso et al., 2010, p. 359).

Los antecedentes teóricos del proceso de aprendizaje turístico para el presente estudio se centran en la teoría del aprendizaje significativo de Ausubel y la teoría del aprendizaje por descubrimiento de Bruner, consecuentes con el paradigma psico-pedagógico cognitivo (tabla 1). El estudio se adapta a las exigencias del Modelo Educativo Constructivista de la Universidad Autónoma de Ciudad Juárez (figura 1), en el que se establecen como principios educativos con relación al alumno: 
a) El alumno es responsable último de su propio proceso de aprendizaje.

b) El sujeto debe jugar un papel activo en su aprendizaje.

c) El aprendizaje es un proceso de construcción del conocimiento y la enseñanza es un apoyo al proceso de construcción social del mismo.

d) Los conocimientos son construidos por los sujetos que se apropian de ellos mediante el lenguaje y la actividad.

Tabla 1.- Matriz cruzada de las situaciones de aprendizaje seleccionadas

\begin{tabular}{|l|l|l|}
\hline $\begin{array}{l}\text { Situaciones de } \\
\text { aprendizaje }\end{array}$ & $\begin{array}{l}\text { Aprendizaje por } \\
\text { recepción }\end{array}$ & $\begin{array}{l}\text { Aprendizaje por } \\
\text { descubrimiento }\end{array}$ \\
\hline $\begin{array}{l}\text { Aprendizaje por } \\
\text { repetición }\end{array}$ & $\begin{array}{l}\text { Recepción } \\
\text { repetitiva }\end{array}$ & $\begin{array}{l}\text { Descubrimiento } \\
\text { repetitivo }\end{array}$ \\
\hline $\begin{array}{l}\text { Aprendizaje } \\
\text { significativo }\end{array}$ & $\begin{array}{l}\text { Recepción } \\
\text { significativa }\end{array}$ & $\begin{array}{l}\text { Descubrimiento } \\
\text { significativo: } \\
\text { guiado y autónomo. }\end{array}$ \\
\hline
\end{tabular}

Elaboración propia basada en Ausubel, s/a; Frida et al, 2002

Al mismo tiempo se reconoce que el rol del profesor y el estudiante deberá estar caracterizado por los aspectos siguientes:

1. El profesor es guía, planea y construye situaciones de aprendizaje, facilita la construcción del aprendizaje, es corresponsable, facilita el proceso, y asesora.

2. El alumno es corresponsable, estratégico, activo, propositivo, autónomo, comprometido; auto controla para planear, realizar y modificar acciones para el aprendizaje.

Para el desarrollo de la actividad orientada al quehacer de los estudiantes se utilizan las 5 dimensiones del aprendizaje propuestas por T. Chan (Chan, 2000).

1) Problematización-disposición. Creación de las condiciones favorables para el desarrollo de la actividad de terreno.

2) Adquisición y organización del conocimiento. Aprenden a integrar la información relativa al tema de manera significativa. Se diferencia la conceptualización de la actividad durante tres fases: antes, durante y después de la visita.

3) Procesamiento de la información. Se basa en el desarrollo de operaciones mentales como la comparación y clasificación. 
4) Aplicación de la información a la solución del caso real al que se enfrentan durante la actividad de terreno en función de lo cual resuelven problemas y toman decisiones e investigan.

5) Conciencia del proceso de aprendizaje- Autoevaluación.

Figura 1.- Sustentos teóricos de la propuesta de aprendizaje turístico descubrimiento significativo mediante los viajes de estudio

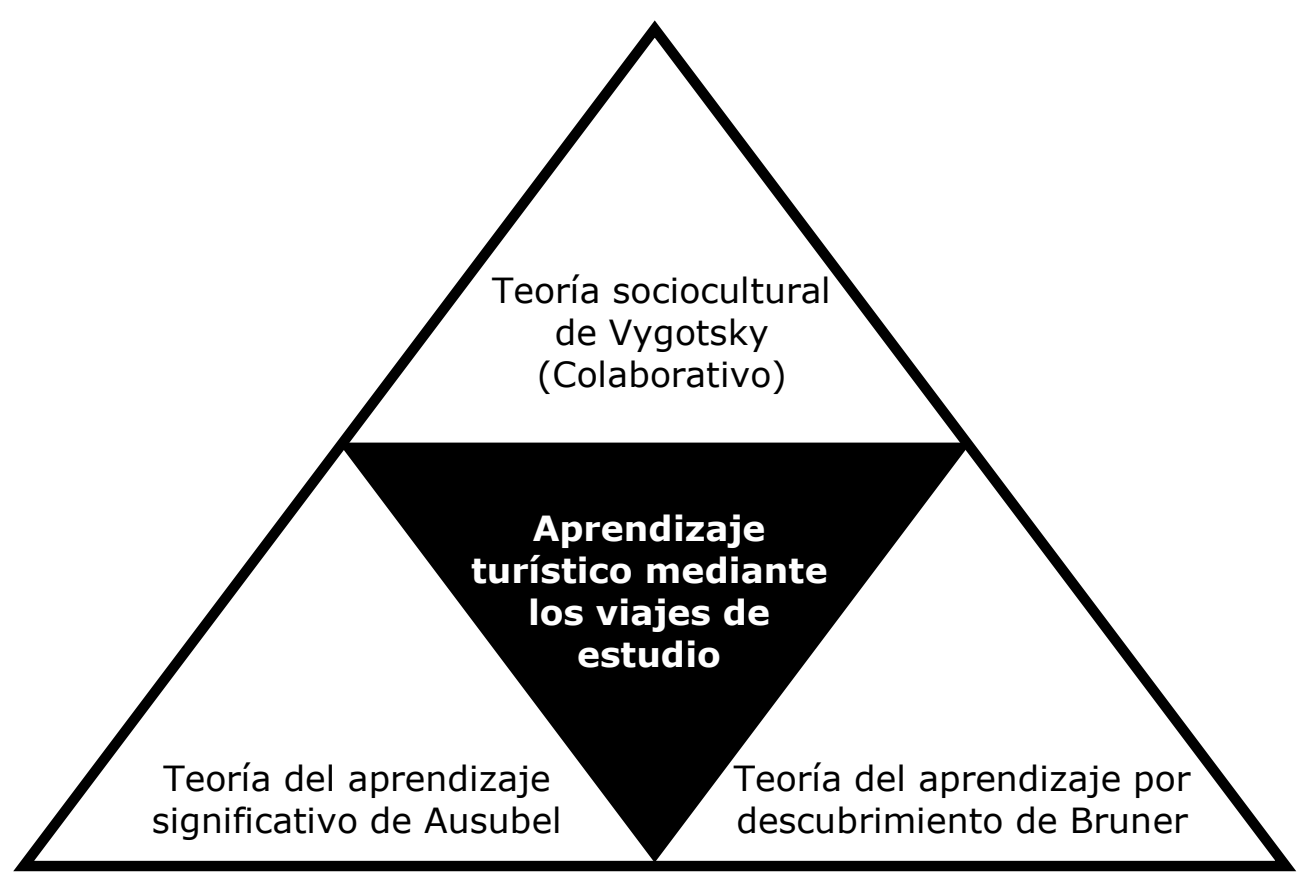

Elaboración propia, basado en Modelo Educativo UACJ, PIME, 2012

Interpretación teórica de los viajes académicos de estudio

Según el antropólogo Sandoval Forero: "Un aspecto característico del quehacer de las disciplinas sociales es el trabajo de campo, el cual se ha constituido en la forma privilegiada de acercarse a la realidad, aunque es muy escaso lo que se ha escrito en torno a su práctica y reflexión en el contexto teórico, metodológico y técnico" (Sandoval, 2006, p. 2). Asimismo señala que: "En los últimos cincuenta años del siglo XX, estudiosos de diferentes disciplinas de la ciencia social y humanística como la antropología, historia, sociología, economía, geografía, psicología, ciencia política y comunicación, han recurrido para sus investigaciones, estudio y formación académica al trabajo de campo" (Sandoval, 2006, p. 7). En este espacio académico investigativo cabe incorporar también el estudio del turismo como fenómeno socio-económico y territorial-ambiental complejo. 
Cabe mencionar que frecuentemente en la literatura y en la práctica pedagógica de los diferentes niveles de formación para conceptualizar este tipo de actividad se utilizan términos tales como trabajo de campo, excursión docente, práctica de campo, prácticas académicas de campo, estudio de la localidad, caminata, expedición, visitas guiadas, paseo, viaje de estudio, viajes de movilidad e intercambio estudiantil, recorrido, entre otros. Seguidamente se revisan tres términos de los más utilizados con el propósito de comprender su definición y los rasgos comunes que permitan establecer regularidades para su implementación. Estas variables conceptuales a comentar son los viajes de estudio, trabajo de campo y práctica de campo.

En correspondencia con la conceptualización relativa a los viajes de estudio de AGTER, se define que: "Un viaje de estudio o taller itinerante se compone de una serie de visitas de terreno articuladas con una temática global precisa... reúne un grupo de personas, que están acompañadas por facilitadores, quienes organizan y guían las visitas de terreno. Es el conjunto de las visitas que debe ser coherente y tener un significado en relación con la temática del viaje. Las visitas pueden también completarse con presentaciones más teóricas, que apuntan a ubicar esta actividad en su contexto histórico, político, económico, social o cultural" (Jamart, 2007, p. 1). El objetivo es provocar una reflexión de los participantes sobre la temática, la realidad del lugar visitado y su propia realidad.

Según Michel Merlet, "hacer un viaje para ver una realidad muy diferente y organizar la reflexión profunda sobre lo que se está viendo, es mucho mejor que exponer una realidad porque uno escucha las palabras, pero no se imagina cómo son las cosas. El diálogo entre todas las personas permite generar un proceso de aprendizaje y un conocimiento sobre lo que está viendo y descubriendo hasta llegar a reflexionar sobre su propia realidad... y es mucho mejor cuando este descubrimiento de otras realidades no se hace solo, sino junto a otras personas que tienen otras experiencias" (Bombino et al., 2012, p. 16).

En particular, el "trabajo de campo es entendido como el acercamiento a la realidad que se pretende estudiar; consiste en acudir directamente al sujeto/objeto de estudio, al dato vivo, a los hechos, para entender la situación y dinámica en que se desarrolla. La información y los datos obtenidos directamente por el investigador se pueden adquirir en infinidad de lugares, sean rurales o urbanos, según lo que se necesite examinar” (Sandoval, 2006, p. 13). 
Al mismo tiempo, se considera que la práctica de campo comprende "aquellas visitas, paseos o caminatas que realizamos con un objetivo didáctico bien definido y que nos permite estudiar objetos o fenómenos de la naturaleza, de la producción o de la sociedad en general, que sin sustituir a la clase como la forma fundamental de organización de este proceso, ofrece múltiples ventajas y se enlaza perfectamente a la misma" (EcuRed, 2015).

Según un grupo de sociólogos de la Universidad de Castilla La Mancha, la práctica de campo es un proceso en el que el estudiante investiga (utilizando técnicas) directamente (de primera mano) y de forma presencial, sumergiéndose (sin perjuicios) en la realidad de un problema, necesidad, etcétera, con el fin de conocerla de forma exhaustiva, para diseñar y llevar a cabo una posterior intervención (Gutiérrez et al., 2006).

En los Lineamientos de la Universidad Autónoma del Estado de México, se reconoce que las Prácticas Académicas de Campo son una actividad académica que se realiza fuera de las instalaciones universitarias, cuyo propósito es ampliar los conocimientos y habilidades adquiridas en forma teórica y experimental. Su finalidad es profundizar y complementar los conocimientos adquiridos en el aula conforme a los contenidos de los planes y programas de estudio (artículo 2). Su objetivo asimismo es coadyuvar al proceso de enseñanza-aprendizaje, desarrollar habilidades y reforzar los conocimientos de los alumnos, así como fomentar el conocimiento a través de la investigación aplicada (artículo 3). Integra 4 modalidades que son (artículo 5): visita guiada; visita de observación; práctica de campo; y participación en eventos científicos (UAEM, 2015).

De acuerdo con los Lineamientos Generales para la Realización de las Prácticas de Campo de la UNAM, esta es una actividad o conjunto de actividades que se llevan a cabo fuera de las instalaciones de las entidades o dependencias donde se encuentren inscritos los alumnos y/o estudiantes, con el propósito de ampliar los conocimientos y habilidades adquiridos en el salón de clases. Por su carácter académico y su relación con los planes de estudio, pueden ser prácticas de campo obligatorias curriculares o prácticas de campo no obligatorias o extracurriculares (UNAM, 2012).

$\mathrm{Al}$ respecto, la UNAM (2012) clasifica las prácticas de campo obligatorias curriculares en 3 modalidades que son: prácticas de campo y viajes de prácticas (duración mayor a 24 horas); visitas guiadas y de observación (duración no mayor a 24 horas), y clases fuera de aulas y ejercicios (duración no mayor a 12 horas). Las prácticas de campo no obligatorias o extracurriculares son salidas no directamente relacionadas con los requisitos curriculares del plan 
y programas de estudio, y tienen la finalidad de ampliar el conocimiento y la cultura de los alumnos o estudiantes. Se desarrollan en 3 modalidades que son: asistencia a concursos y actividades deportivas (competencias); asistencia a congresos, foros académicos, seminarios, intercambio y estancia académica; y asistencia a actos artísticos y culturales.

En diferentes universidades e instituciones universitarias, las prácticas de campo podrán también realizarse en el extranjero, ocasión en la que, por ejemplo, según el Reglamento de Prácticas de Campo de la ENAH, será necesario presentar carta de invitación del proyecto o localidad en donde se realizará la práctica, copia del documento oficial que autorice el ingreso al país (visa y/o pasaporte) y copia del comprobante médico que garantice la cobertura en el extranjero (ENAH, 2015), entre otros requerimientos, según sea el caso.

Las ideas básicas contenidas en las anteriores definiciones permiten comprender los viajes académicos de estudio a través de los rasgos siguientes:

1) Forma de organización del proceso de enseñanza aprendizaje, de carácter docente e investigativa aplicada, que se desarrolla mediante visitas/prácticas de campo/terreno en un contexto específico, fuera de las instalaciones universitarias y con un objetivo didáctico definido.

2) Articulación a una temática determinada relacionada con los planes y programas de estudio de la especialidad, las cuales pueden ser obligatorias o curriculares y no obligatorias o extracurriculares.

3) Formación/ampliación de conocimientos significativos y por descubrimiento basado en la reflexión, la socialización y el aprendizaje grupal.

4) Desarrollo/fortalecimiento de habilidades prácticas y capacidades profesionales.

5) Comprensión dinámica e in situ de objetos y fenómenos de la naturaleza, de la sociedad y/o de la producción.

6) Se puede realizar mediante diferentes modalidades, tales como visita de observación, actividades de interpretación, senderos auto guiados, recorridos contemplativos, entre otras. Se desarrollan con diferente duración, por lo que pueden ser de medio día (duración no mayor de 6 horas), del día completo (duración menor a 24 horas), de fines de semana, y de 1 semana o más (duración mayor de 24 horas), en cuyos casos se requiere de alojamiento. Pueden tener un alcance local, regional, o internacional. 
Este tipo de actividad académica e investigativa es de gran importancia, ya que contribuye a la "preparación para el aprendizaje de la profesión y debe estar dirigida a potenciar la aproximación progresiva de los estudiantes - desde el primer año - a los problemas de la profesión, factor determinante para lograr un profesional preparado" (EcuRed, 2015) de forma vivencial y participativa en el ámbito del turismo. Por tal razón, es comprensible que debe ser una actividad sistemática, integradora y contextualizada, en la que se enfrente a los estudiantes con situaciones prácticas demostrativas de aprendizaje. Esto facilitará que los contenidos sean mejor asimilados y/o consolidados, permitiendo la generación e intercambio de información y experiencias.

El maestro o facilitador debe conocer las posibilidades de aplicación de cada una de las modalidades de los viajes académicos de estudio o prácticas de campo, de forma tal que se seleccione la más apropiada para el tratamiento de los contenidos y el desarrollo de habilidades prácticas y capacidades profesionales. Será oportuna la organización de grupos de trabajo pequeños, la creación de dinámicas favorables entre los participantes, la guía y supervisión de los profesores encargados, así como la creación de comisiones para la planificación, desarrollo y control del trabajo. Deberá lograrse que los participantes estén comprometidos, implicados, involucrados y responsabilizados con el cumplimiento de los objetivos y actividades del viaje de inicio a fin (Jamart, 2007).

\section{Método}

La metodología utilizada se basó en la aplicación del enfoque inductivo. El estudio corresponde a una investigación de tipología aplicada; causal/explicativa en correspondencia con la solución a las interrogantes de investigación que fueron planteadas; cuali-cuantitativa; transversal; y de carácter experimental; en la que se utilizan fuentes de información mixta. La unidad de análisis y de muestreo corresponde a dos grupos de estudiantes de la Licenciatura en Turismo del campus Ciudad Universitaria (CU) de la Universidad Autónoma de Ciudad Juárez. El área de estudio correspondió a Playa Puerto Peñasco, Sonora, escenario donde se realizó la actividad práctica de terreno para favorecer el desarrollo de aprendizajes significativos por descubrimiento mediante los viajes de estudio.

Se utilizaron métodos de los niveles empírico, teórico y estadístico-matemáticos. Los métodos empíricos permitieron la intervención, registro, medición, análisis e interpretación de la realidad mediante la observación; análisis de documentos; encuestas y entrevistas; y criterio de 
expertos/especialistas. Los métodos teóricos facilitaron la construcción y desarrollo de argumentos correspondientes a la teoría científica, las regularidades y características esenciales de los fenómenos, utilizando los métodos analítico-sintético; inductivo-deductivo; abstracciónconcreción; y la modelación. Los métodos estadístico-matemáticos y computacionales permitieron asentar datos recolectados, realizar procesamiento digital y arribar a conclusiones. En vínculo a estos métodos se aplicaron diferentes técnicas y procedimientos conceptuales; descriptivos; y métricos, tanto cualitativos como cuantitativos para la localización, discriminación y selección de datos, extracción de la información que se requiere de las fuentes seleccionadas, procesamiento, medición y análisis de los datos según criterios de medida y escala de medición.

Para estudiar el impacto del aprendizaje turístico por descubrimiento significativo mediante los viajes académicos de estudio se realizó un experimento pedagógico cuya operacionalización se basó en la identificación de: población objeto de estudio y tipo de muestra; instrumento de recolección de datos: escala de medición; procedimiento; y análisis de los datos. Se desarrolló un experimento pedagógico sucesional proyectado en el que el grupo experimental coincide con el de control, el cual fue sometido al estímulo experimental. El grupo experimental/control desarrolló una práctica de terreno con una duración de 3 días para el estudio vivencial del tema "Producción y estructuración del espacio turístico en Playa Peñasco, México".

El propósito general del viaje fue implementar el enfoque geoespacial al estudio de destinos turísticos mediante instrumentos sencillos de gestión del espacio local. Para cumplimentarlo se enunció como actividad la aplicación práctica del enfoque geoespacial al estudio de un destino turístico litoral de playa mediante la interpretación del proceso de producción del espacio turístico, el reconocimiento del modelo de implantación geoespacial, el inventario y evaluación de recursos turísticos de base territorial y el análisis del esquema de estructuración del espacio turístico local. 


\section{Resultados y discusión}

Componente práctico formativo del Programa de Licenciatura en Turismo de la UACJ: viajes académicos de estudio en las modalidades de visitas y recorridos curriculares

El desarrollo de actividades de terreno en el Programa de Licenciatura en Turismo de la Universidad Autónoma de Ciudad Juárez ha estado relacionado con el componente práctico formativo. La reestructuración del Programa de Prácticas Profesionales (tabla 2) incorpora 4 tipos o modalidades de prácticas que se adaptan a las tendencias actuales de formación profesional, mediante un proceso escalonado, sistemático y progresivo de conocimientos, habilidades y capacidades. Estas son:

Tabla 2.- Estructura organizacional del sistema de Prácticas Profesionales de Turismo UACJ.

\begin{tabular}{|c|c|c|c|}
\hline & Nivel Principiante & $\begin{array}{c}\text { Nivel } \\
\text { Intermedio } \\
\end{array}$ & $\begin{array}{c}\text { Nivel } \\
\text { Avanzado }\end{array}$ \\
\hline $\begin{array}{l}\text { Práctica } \\
\text { inducción. } \\
(42 \mathrm{hs})\end{array}$ & $\begin{array}{l}\text { Visita grupal a un } \\
\text { restaurante }(5 \mathrm{hs}) \text {. } \\
\text { Visita grupal a un } \\
\text { hotel ( } 5 \mathrm{hs}) \text {. } \\
\text { Recorrido turístico } \\
\text { por la ciudad ( } \\
\text { hs). } \\
\text { Visita a una AAVV } \\
(5 \mathrm{hs}) \text {. }\end{array}$ & $\begin{array}{l}\text { Visita ecoturística } \\
(20 \mathrm{hs}) . \quad[\text { En la } \\
\text { materia Turismo y } \\
\text { Medio Ambiente.] }\end{array}$ & \\
\hline $\begin{array}{l}\text { Práctica } \\
\text { aproximación. } \\
(140 \mathrm{hs})\end{array}$ & $\begin{array}{l}\text { Trabajo de } \\
\text { investigación } \\
\text { aplicada }(20 \mathrm{hs}) \text {. }\end{array}$ & $\begin{array}{l}\text { Trabajo de } \\
\text { investigación } \\
\text { aplicada }(40 \mathrm{hs}) .\end{array}$ & 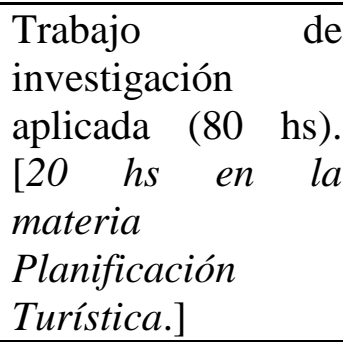 \\
\hline $\begin{array}{l}\text { Práctica } \\
\text { simulación. } \\
(58 \mathrm{hs})\end{array}$ & $\begin{array}{l}\text { Laboratorio } \\
\text { gastronómico } \\
\text { hs }) \text { (20 } \\
\text { Laboratorio } \\
\text { cómputo de } \\
\text { desk (18 hs). }\end{array}$ & $\begin{array}{l}\text { Laboratorio } \\
\text { gastronómico } \\
\text { hs }) . \\
\text { Organización } \\
\text { evento }(10 \mathrm{hs}) .\end{array}$ & \\
\hline $\begin{array}{l}\text { Práctica de } \\
\text { profesionalización. } \\
(960 \mathrm{hs})\end{array}$ & & \multicolumn{2}{|c|}{ 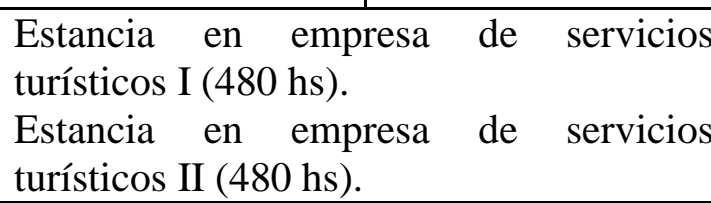 } \\
\hline
\end{tabular}

Fuente: elaboración propia basada en Plan Curricular 2014, Programa de Licenciatura en

Turismo, ICSA, UACJ. Versión 4 de septiembre de 2013. 
1) Inducción (anteriormente denominadas de Familiarización): con un total de 42 horas.

2) Aproximación (anteriormente denominadas de Observación): con un total de 140 horas.

3) Simulación: con un total de 58 horas.

4) Profesionalización (anteriormente denominadas de Especialización): con un total de 960 horas.

En tal sentido, las Prácticas Profesionales se redimensionan integrando en niveles formativos progresivos las dimensiones cognitivo instrumental, afectivo motivacional, actitudinal (sobre una sólida base de valores) y comportamental. Mediante esta última es posible reforzar la formación de comportamientos responsables ante cada una de las áreas en que deberán desempeñarse los estudiantes una vez egresados.

Del total de 1200 horas de prácticas profesionales, solo 42 horas corresponden a viajes académicos de estudio en las variantes de visitas y recorridos curriculares, las cuales son desarrolladas en la modalidad de práctica de inducción. El resto se relaciona con trabajos de investigación aplicada (que puede incluir actividades de terreno), laboratorios y estancia en empresas o instituciones públicas o privadas en que se prestan servicios turísticos; nótese que no se desarrollan viajes académicos de estudios curriculares en las modalidades de prácticas de aproximación, simulación y profesionalización.

Esta nueva reestructuración de las Prácticas Profesionales en la UACJ contribuirá en diferentes horizontes temporales futuros a:

1) Consolidación de los viajes académicos de estudio como forma de organización del Proceso Docente Educativo, mediante la cual se alcanza una favorable vinculación del alumno con el desarrollo de actividades de terreno.

2) Perfeccionamiento permanente del vínculo entre la formación práctica y el perfil del egresado.

3) Desarrollo de aprendizajes por descubrimiento significativo a través de las visitas de terreno a diferentes destinos turísticos.

4) Compromiso por parte de los estudiantes con la sustentabilidad y protección del medio ambiente de uso turístico.

5) Trabajo colaborativo y desarrollo de habilidades de trabajo práctico. 
6) Evaluación formativa del desempeño durante el desarrollo de las actividades prácticas de campo.

7) Elaboración de informe final de las visitas de estudio con enfoque investigativo, utilizando una bitácora de experiencias en la que se autoevalúan los logros alcanzados y los retos para próximas actividades.

\section{Propuesta de un instrumento para el desarrollo de viajes académicos de estudio extracurriculares}

Basado en el diagnóstico del plan de estudio de la Licenciatura en Turismo de la UACJ se propone la construcción e implementación de un instrumento y de los procedimientos metodológicos para favorecer el aprendizaje turístico por descubrimiento significativo mediante los viajes académicos de estudio extracurriculares. Este se formula en respuesta a las oportunidades que ofrece la materia de Planificación Turística correspondiente al nivel avanzado de la currícula, y da respuesta a los contenidos prácticos del Tema 2 "Planeación física y Ordenamiento Territorial del Turismo", en lo relativo al Espacio Turístico Litoral. El instrumento construido se sintetiza a continuación.

Tema. Producción y estructuración del espacio turístico como base para la sustentabilidad. Caso Playa Peñasco, México.

Tiempo de duración: 3 días.

Objetivo. Implementar el enfoque geoespacial al estudio de destinos turísticos litorales del tipo playa mediante instrumentos sencillos de gestión del espacio local como base para alcanzar la consolidación de modelos de desarrollo turístico sustentables.

Enunciado de la actividad. Aplicación práctica del enfoque geoespacial al estudio de un destino turístico litoral de playa mediante la interpretación del proceso de producción del espacio turístico, el reconocimiento del modelo de implantación geoespacial, el inventario y evaluación de recursos turísticos de base territorial y el análisis del esquema de estructuración del espacio turístico local. 
Actividades de terreno:

1. Interpreta el proceso de producción del espacio turístico según fuentes históricas, evidencias y entrevistas con los residentes locales.

2. Reconoce el modelo de implantación geoespacial del turismo en el contexto regional según tipología del análisis deductivo. Identifica las características observables que justifican cada tipo.

3. Realiza el inventario turístico del recurso de playa según ficha de inventario.

Platilla inventario del recurso turístico playa.

a) Localización. Ubicación geográfica y distancia de puntos de interés cercanos.

b) Características generales. Tipología y clasificación del recurso; forma de la playa; estructura general de la playa; tipo de arena por su origen; calidad de la arena; color de la arena; granulometría de la arena en la línea de la costa; transparencia del agua; color del agua; evidencias de rompimiento; oleaje promedio; vegetación litoral y marina; características del sistema costero detrás de la duna litoral; fauna perjudicial; actividad antrópica; naufragios, pesca deportiva, corales, etcétera.

c) Dimensiones de la playa (en metros). Longitud total de la playa; ancho medio de la playa emergida; superficie de la playa; distancia promedio entre la profundidad de $1.8 \mathrm{~m}$ y la línea de la costa; ancho medio de la franja del área de sol; ancho medio de la franja del área de la sombra; longitud aprovechable de la playa (por arrecifes, mala calidad de la playa u otro); capacidad de carga turística.

d) Planta turística. Instalaciones de alojamiento turístico hoteleros existentes; número de habitaciones y capacidad, categoría; restaurantes, cafeterías y bares; baños públicos; piscinas; miradores turísticos; agencias de viajes, oficinas de información turística, otras.

e) Accesibilidad, conectividad y comunicaciones. Carretera; terracería, sendero, muelle, atracadero, vía férrea; aeropuerto; pista de aterrizaje; teléfonos y correo, bancos, etcétera.

f) Abastecimiento de agua para uso general. Localización de las fuentes de abasto; conducción del agua; volumen disponible; capacidad de almacenaje; calidad del agua; energía eléctrica.

g) Estado de calidad ambiental. Problemas ambientales observables: erosión de la playa; alteraciones del escurrimiento pluvial; pérdida de la vegetación y la fauna; fuentes y tipo de 
contaminación ambiental (basura, albañales, humo, polvo, ruido, vibraciones, gases nocivos, olores desagradables, derrames de petróleo, fumigación, otros agentes químicos o físicos).

h) Valoración General. Potencialidades; restricciones; observaciones.

4. Realiza la evaluación del recurso turístico playa según método de valoración cualitativa por puntos en una escala de 3 rangos ( 1 baja, 2 media, 3 alta). Para ello se utilizarán:

Indicadores de valor funcional de uso turístico: Unicidad: valor que adquiere un producto por el hecho de ser único; Valor intrínseco: valor que tiene un producto dentro de su categoría; Carácter local: valor que se le otorga a un producto por ser típico o característico; Concentración de la oferta: valor derivado de la concentración de productos para realizar otras actividades turísticas dentro de la misma zona; Notoriedad: grado de conocimiento del producto, tanto dentro del país como a nivel internacional.

Indicadores para la evaluación estética: Contraste; Visibilidad; Gama tonal de colores; Movimientos y sonidos; Elementos pintorescos; Exotismo; Potencial de visibilidad: abertura, cuenca visual, efectos de perspectivas.

Indicadores para la evaluación ambiental: Tipicidad o unicidad; Rareza; Singularidad; Naturalidad; Fragilidad; Valores ecológicos; Valores añadidos; Diversidad

5. Analiza el esquema de estructura de espacio turístico según correspondencia entre las zonas morfológicas y funcionales del perfil de playa.

Identificación de modalidades turísticas vinculadas al espacio. Se tendrá en cuenta: Zona de baño y actividades náuticas: actividades turísticas recreativas en la zona de la playa sumergida; otras observaciones; Zona de circulación: objetos de obra y actividades turísticas recreativas; Zona de soleamiento: objetos de obra y actividades turísticas recreativas; Zona de sombra: tipo se sombra (natural/artificial), diseño, objetos de obra y actividades turísticas recreativas; Zona de transición a la playa: objetos de obra y actividades turísticas recreativas; Zona construida: tipología y modelo constructivo; densidad de ocupación; accesos peatonales a la playa; otras observaciones.

Representa de forma esquemática el perfil morfológico y funcional en un punto seleccionado de la playa.

Evaluación de la actividad: participación en las actividades diarias y aportación de ideas. Solución de las tareas propuesta de forma individual y grupal. Elaboración del informe final de la visita. Participación activa en el taller de debate de los resultados de la visita. 
Conocimientos turísticos curriculares que sirven de soporte teórico al desarrollo al viaje académico de estudio en la materia de Planificación Turística

El contenido corresponde a "El espacio turístico litoral. Planificación del espacio turístico litoral", en el que se abordan como elementos temáticos:

1) El espacio litoral. Tipos de espacios litorales. Recursos y atractivos litorales.

2) Modalidades turísticas asociadas al litoral: sol y playa, náutica, buceo, pesca deportiva, crucero y yate.

3) Características de los mercados que demandan modalidades de turismo litoral.

4) Playas de arenas. Formas de las playas. Partes de la playa. Zonificación morfológica. Zonificación funcional.

5) Problemas del uso turístico del litoral.

Se trata de una temática eminentemente práctica que requiere de actividades de campo, bien sea en áreas próximas a la universidad o en polígonos donde el desarrollo del turismo lo permita. Durante el tratamiento de estos contenidos se aplicaron técnicas de análisis regional, en las que se concedió un importante rol a la cartografía como técnica de representación y base de análisis. La misma se fundamentó en la lectura de mapas y la representación cartográfica y análisis geoespacial de destinos turísticos representativos del fenómeno estudiado.

Los aspectos considerados en la planeación y ordenamiento territorial del espacio turístico fueron:

1. Conceptualización del desarrollo turístico integrado del destino. Producto turístico. Corresponde al modelo de desarrollo turístico implementado y el tipo y nivel de desarrollo del producto turístico (figura 2). 
Figura 2. Componentes integrados para el estudio del producto turístico

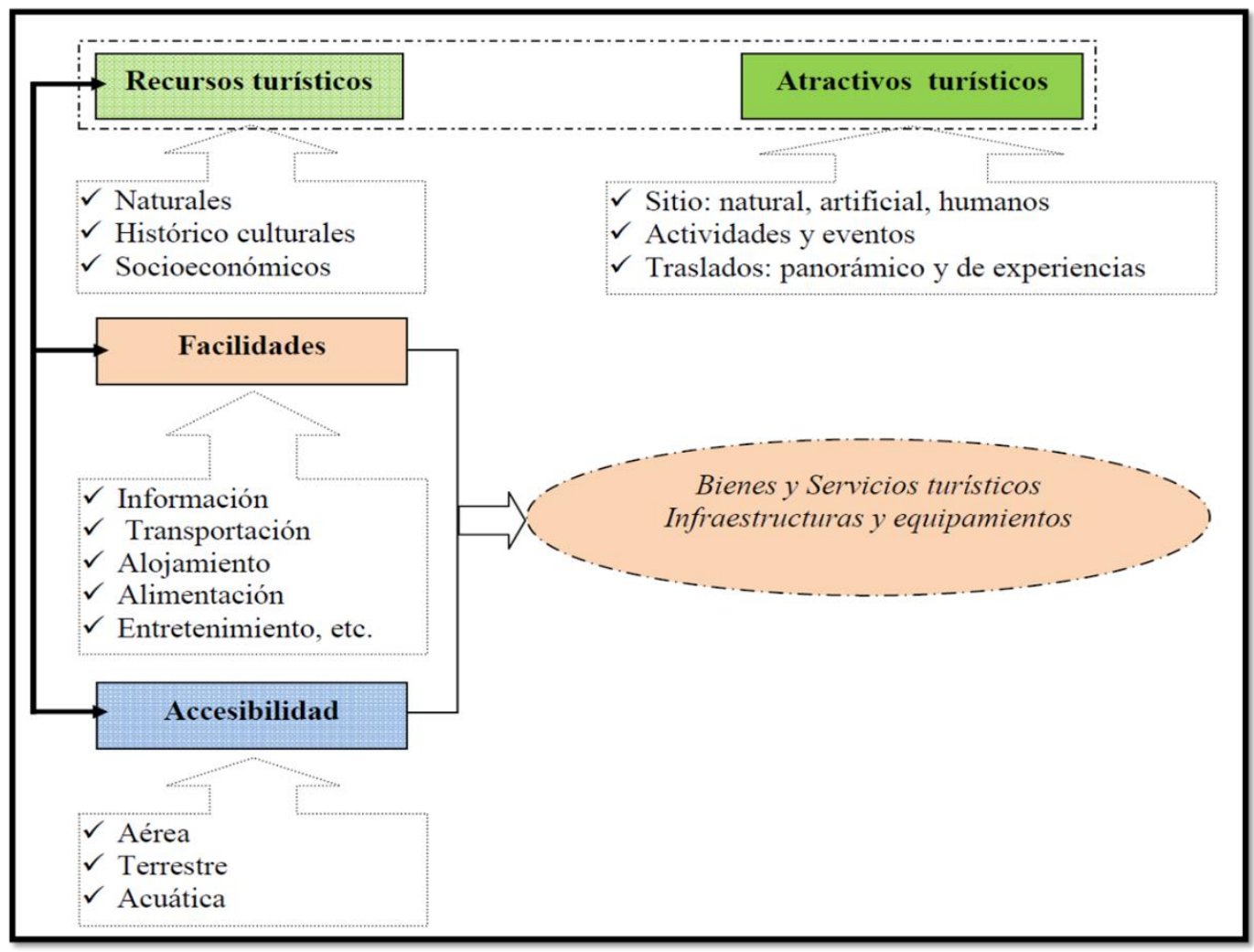

Fuente: elaboración propia.

2. Tipología de los espacios a planear. Atendió a la tipología del destino turístico, considerando las diferencias entre espacios turísticos naturales, litorales, rurales y urbanos. Fueron consideradas las etapas del proceso de producción del espacio turístico (figura 3).

3. Determinación del potencial turístico. Se consideró el potencial turístico según: valor turístico (funcional, ambiental, estético, simbólico) de los recursos - atractivos, su lugar de emplazamiento y el entorno, lo cual es indicativo de la capacidad turístico-productiva disponible para satisfacer las necesidades del mercado. Es expresión de la aptitud o vocación de uso turístico, en función de lo cual debe considerarse la compatibilidad entre la capacidad de carga y las condiciones de respuestas derivadas de su uso. Se implementó el enfoque representado en la figura 3 para la comprensión del proceso de producción del espacio turístico objeto de estudio. 
Figura 4. Proceso de Producción del Espacio Turístico

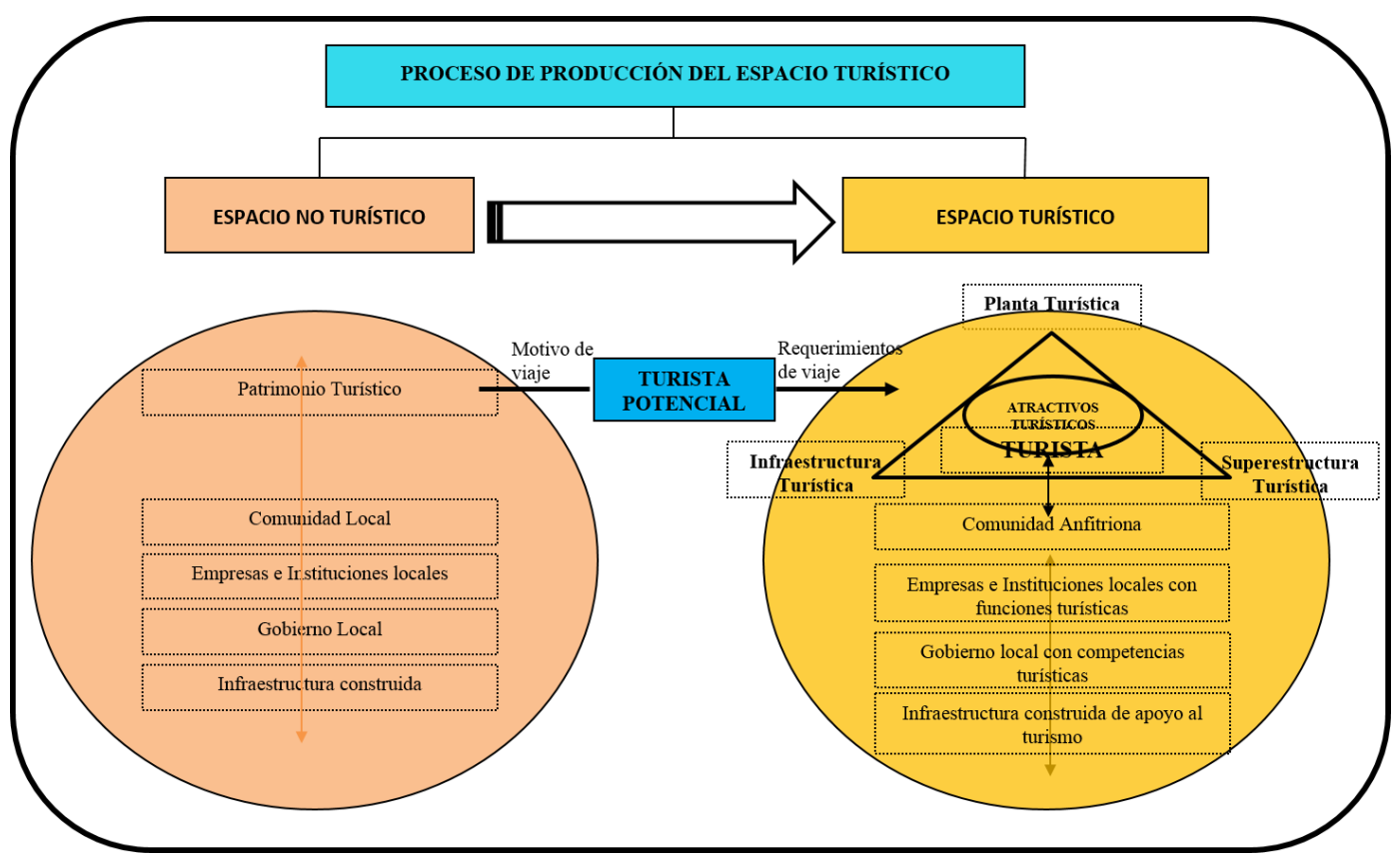

Fuente: elaboración propia.

Se consideraron los conceptos de Potencial Turístico como la capacidad que tienen los productos turísticos para satisfacer los gustos y preferencias actuales de los visitantes (PST, SECTUR, 2013-2018) y Vulnerabilidad como el nivel en el que un sistema es susceptible, o no es capaz de soportar los efectos adversos; la vulnerabilidad está en función del carácter, magnitud y velocidad de la variación a la que se encuentra expuesto un sistema, su sensibilidad y su capacidad de adaptación (PST, SECTUR, 2013-2018).

Las etapas del procedimiento metodológico para la determinación del potencial turístico asumidas fueron:

A. Inventario de recursos atractivos turísticos. Uso actual y potencial. Facilidades (servicios y equipamientos) y accesibilidad.

B. Caracterización de los recursos atractivos.

C. Evaluación de potencialidades turístico-recreativas (aptitud o vocación turística v/s restricciones).

Para el inventario de recursos atractivos turísticos se utilizó la técnica del inventario de recursos turísticos, considerando: Tipología y clasificación; Localización. Accesibilidad y accesos; Criterio toponímico; Descripción de los atributos; Observaciones.

Los métodos de inventario estudiados fueron: Listas de control; Matrices; Diagramas de flujo. 
La caracterización de los recursos atractivos se desarrolló siguiendo un plan de caracterización. La evaluación de la aptitud o vocación turística del recurso/atractivo atendió a los valores de referencia siguientes:

1) Valor funcional de uso turístico determinado por su posible aprovechamiento (valores tangibles e intangibles).

2) Valor estético según belleza natural o artificial.

3) Valor simbólico o representativo según el efecto de emoción/sensación que transmite su uso.

4) Valor ambiental según características ambientales del recurso y requerimientos de conservación.

5) Valores añadidos según atributos incorporados mediante la actividad del hombre.

Los métodos de evaluación de los recursos turísticos atractivos estudiados fueron: Método de evaluación por puntos; Matrices; Diagramas; Tormentas de ideas; Modelación.

4. Estudio de capacidad de carga y zonificación funcional. Se consideró la capacidad de carga como el número de visitantes y grado de desarrollo óptimo que no genere efectos adversos. Integra las características de los recursos y de los turistas potenciales. La zonificación funcional del potencial turístico se conceptualizó como una técnica de la planificación territorial que define tipos de manejos específicos para cada territorio (base técnica y científica), por lo que sugiere usos adecuados para cada territorio.

5. Ordenamiento territorial. Se presentaron los conceptos básicos a utilizar asumiendo el Ordenamiento Turístico del Territorio en México: Instrumento de la política turística bajo el enfoque social, ambiental y territorial, cuya finalidad es conocer e inducir el uso de suelo y las actividades productivas con el propósito de lograr el aprovechamiento ordenado y sustentable de los recursos turísticos, de conformidad con las disposiciones jurídicas aplicables en materia de medio ambiente y asentamientos humanos (PST, SECTUR, 2013-2018).

De igual forma se analizó el concepto de Centro Integralmente Planeado (CIP): Centro turístico de gran escala e impacto nacional, desarrollado por FONATUR, siguiendo los lineamientos establecidos en un Plan Maestro con horizonte de planeación de largo plazo, que considera de forma integral los aspectos turísticos, urbanos, ambientales, sociales y económicos, y que requiere para su puesta en marcha de importantes acciones de dotación o complementación 
de infraestructura regional. Un CIP podrá desarrollarse como un proyecto unitario o con base a dos o más Proyectos Turísticos Integrales (PST, SECTUR, 2013-2018).

Finalmente, se estudió el Sistema de Evaluación de Destinos Turísticos en México (SEDET), el cual representa una evaluación integral aplicada a los destinos turísticos y que comprende investigación de gabinete, entrevistas y reuniones de trabajo con empresarios y autoridades locales y visitas a los atractivos del destino. Al finalizar, se genera un reporte con recomendaciones para responsables públicos y privados que maximicen beneficios, calidad de vida de los pobladores y conserven el patrimonio ecológico y cultural local (PST, SECTUR, 2013-2018).

\section{Experiencias de aprendizaje turístico por descubrimiento significativo mediante los} viajes académicos de estudio

La etapa experimental se basó en el desarrollo del viaje académico de estudio mediante la secuencia de tres etapas abordadas de forma sistemática, las que comprenden las actividades realizadas por los estudiantes, antes, durante y después de la actividad de terreno, estas son:

1) Pre viaje o antes del comienzo del viaje. Se realizó la planificación y organización del viaje y su logística, con énfasis en la determinación del lugar de visita y la estación de campo a utilizar (en algunas especialidades suelen utilizar casas de la propia comunidad), la selección de los alumnos que participarían y el programa a desarrollar con las tareas apropiadas de estudio. Comprendió la autopreparación teórica por parte de los alumnos con respecto a los contenidos a tratar, según la guía de auto preparación presentada por el maestro o facilitador y la elaboración de los instrumentos a implementar.

2) Durante el viaje o desarrollo de la práctica de campo. La ejecución del viaje de estudio se realizó en correspondencia con el itinerario de viaje programado. Cada sesión comenzó con una breve reunión para la organización del trabajo del día, la asignación de las tareas de estudio y las técnicas e instrumentos a utilizar, la realización propiamente de la visita de terreno, el trabajo de gabinete posterior para el tratamiento de la información recopilada y los talleres de reflexión y debate sobre la jornada diaria, los cuales fueron grabados con el fin de no perder información valiosa. 
3) Fueron utilizadas variadas técnicas de trabajo de campo, como la observación participante, entrevistas, encuesta, entre otras. Al mismo tiempo, se implementaron instrumentos de trabajo de campo como la libreta o registro de campo, diario de campo, guía de observación, cuestionarios, guía de entrevista, portafolios, plantillas o fichas de registros de campo, guías de trabajo de campo, mapas y croquis, fichas de trabajo de terreno, grabadora, cámara fotográfica y de video, GPS, entre otros.

4) Post viaje o después de realización de la práctica de campo. Integró las reflexiones finales, socialización de experiencias, vivencias y conocimientos, transmisión de las experiencias y aprendizajes. Se basó en la presentación de la memoria o informe del viaje académico de estudio, en el que quedaron sistematizados los nuevos conocimientos adquiridos durante las actividades de terreno, y se describieron las nuevas vivencias y experiencias. Finalmente, los estudiantes realizaron una evaluación de la actividad desarrollada. Esto con el fin de conocer la percepción sobre la actividad y los niveles de satisfacción con respecto a la misma, así como las recomendaciones para el perfeccionamiento continuado de esta actividad.

Estas etapas correspondientes al ciclo de vida del viaje académico de estudio se corresponden directamente con las etapas del experimento pedagógico desarrollado. El mismo incluyó:

1) Fase de constatación de la formación inicial. Consistió en un diagnóstico participativo con base en un conjunto de indicadores relacionados con los ejes temáticos a abordar en la visita de terreno, después de concluir el tema estudiado en clase. Este diagnóstico inicial permitió comprobar el nivel de desarrollo de habilidades prácticas y experiencias con relación al destino turístico y el tema objeto de estudio. De un total de 25 estudiantes participantes, 12 nunca habían estado en la playa, 6 habían ido solo una vez, pero apenas recordaban la experiencia y el resto aunque no con muchas vivencias había estado más de una vez en algún destino turístico litoral y su percepción era la de un turista, no la de un turistólogo o turismólogo.

2) Fase de constatación de formación intermedia. Ejecución de las actividades y acciones que comprende la planificación realizada.

3) Fase de constatación de formación final. Abarca el momento posterior a la realización del viaje y fue desarrollada con el fin de reconocer los efectos producidos por la experiencia vivencial y participativa de que fueron partícipes en correspondencia con el instrumento 
elaborado, la metodología diseñada y las actividades de campo implementadas, así como los instrumentos de evaluación formativa diseñados para el control sistemático de los resultados que fueron alcanzados progresivamente. Esto permitió corroborar los cambios que tuvieron lugar, a través de la observación participante, diálogos con los estudiantes, debates en sesiones de trabajo, entrevistas informales, revisión de actividades realizadas, auto valoración, estudio del producto de la actividad (esquemas, dibujos, plantillas, etcétera). Los resultados fueron analizados en su proyección individual y grupal.

\section{Conclusión}

Las principales lecciones de aprendizajes basadas en la experiencia vivida durante el viaje académico de estudio extracurricular corresponden a:

1. Mediante el proceso de aprendizaje práctico se contribuye a la construcción de nuevos conocimientos turísticos formados y reformulados a través de la experiencia, y al desarrollo de habilidades prácticas, valores y actitudes relativos al turismo, los cuales fortalecen el perfil de egreso de los estudiantes y responden a las necesidades del sector turístico.

2. El aprendizaje turístico por descubrimiento significativo guiado mediante los viajes académicos de estudio genera un proceso activo de interacción entre los estudiantes y el ambiente "litoral de uso turístico", lo cual es de gran importancia para el aprendizaje de la profesión.

3. Los viajes académicos de estudio extracurriculares son una valiosa forma de organización del proceso de enseñanza aprendizaje con enfoque investigativo aplicado in situ, en el que mediante visitas de terreno se aborda una temática específica que permite la formación/ampliación de conocimientos significativos y por descubrimiento basado en la reflexión, la socialización y el aprendizaje grupal, así como el desarrollo/fortalecimiento de habilidades prácticas y capacidades profesionales.

4. Los viajes académicos de estudio extracurriculares contribuyen al compromiso in situ de los estudiantes con la sustentabilidad y protección del medio ambiente de uso turístico; al trabajo colaborativo y desarrollo de habilidades de trabajo práctico; a la evaluación formativa del desempeño durante el desarrollo de las actividades prácticas de campo; y a la elaboración de informe final de las visitas de estudio con enfoque investigativo. 
5. Es recomendable la construcción didáctica de instrumentos y de los procedimientos metodológicos ajustados a los objetivos del viaje académico de estudio y las necesidades del perfil del profesional con el propósito de favorecer el aprendizaje turístico por descubrimiento significativo y garantizar la eficiencia formativa de las actividades realizadas.

6. Se comprueba una estrecha relación entre la formación teórico práctica en clases y la formación práctica durante el viaje académico de estudio extracurricular, lo que refuerza los contenidos turísticos curriculares en la materia Planificación Turística.

7. El tratamiento de los contenidos que facilitan el aprendizaje turístico mediante los viajes académicos de estudio debe ser sistémico, integrando de forma holística las diferentes etapas del ciclo de vida del viaje, es decir, la preparación antes del viaje, la realización de las actividades planificadas durante el viaje y el cierre de las acciones previstas para la etapa posterior al viaje. 


\section{Bibliografía}

Ateljevic I., N. Morgan, and A. Pritchard. (2011). The Critical Turn in Tourism Studies. Creating an Academy of Hope. Routledge. Taylor \& Francis Group Ltd. [recuperado el 28 de agosto de 2015].

Ausubel,

D.

(s/a). Teoría

del

Aprendizaje

Significativo.

http://www.ipprojazz.cl/intranet_profesor/subir_archivo/archivos_subidos/Aprendizaje_signi ficativo.pdf [recuperado el 20 de julio de 2015].

Bombino Y., M. García, D. Echevarría, N. Pérez. (2012). Viajes de estudio: experiencia metodológica para el aprendizaje por contraste. Ruth Casa Editorial. Panamá. http://www.ruthcasaeditorial.org/libroslibres/wpcontent/uploads/2014/02/Aprendizaje_por_contraste.pdf

Chan, T. (2000). Guía para la elaboración de materiales orientados al aprendizaje autogestivo. Innova. Universidad de Guadalajara, México.

EcuRed Conocimientos con todos y para todos. (2015). Prácticas de Campo. Cuba. http://www.ecured.cu/index.php/Pr\%C3\%A1ctica_de_campo [recuperado el 28 de agosto de 2015].

Escuela Nacional de Antropología e Historia /ENAH (2015). Convocatoria prácticas de campo 2015-2. http://www.enah.edu.mx/index.php/invest/pc-enah [recuperado el 28 de agosto de 2015].

Elías, J. (2012). Constructivismo. Curso de Modelo Educativo de la Universidad Autónoma de Ciudad Juárez. Programa de Implementación del modelo educativo (Inédito).

Equipo Institucional del Modelo Educativo UACJ. (2002). Modelo educativo de la Universidad Autónoma de Ciudad Juárez. Visión 2020, segunda edición. Dirección General de Información y Acreditación. Ciudad Juárez, México, 85 pp.

Frida, A., y G. Hernández (editores). (2002). Estrategias Docentes para un Aprendizaje Significativo. Capítulo 2 Constructivismo y Aprendizaje Significativo, segunda edición, McGraw- Hill, México.

Gössling S.; C. Michael; and D. Weaver (2012). Sustainable Tourism Futures. Perspectives on Systems, Restructuring and Innovations. Routledge. Taylor \& Francis Group Ltd.

Gutiérrez, M., M. Maldonado, Á. González, M. Sánchez (2006). Docencia de calidad en las prácticas de campo: experiencia de la EUTS de Cuenca en segundo curso. Universidad de 
Castilla La Mancha. https://papiro.unizar.es/ojs/index.php/ais/article/view/437 [recuperado el 2 de septiembre de 2015].

Jamart, C. (2007). Los viajes de estudio de AGTER. Definición, objetivos y método. Asociación para mejorar la Gobernanza de la Tierra, del Agua y de los Recursos Naturales. http://www.agter.asso.fr/IMG/pdf/Jamart_voyages_d_etudes_es.pdf [recuperado el 28 de agosto de 2015].

Jenkins, I and R. Schröder. (2013). Sustainability in Tourism. Springer Gabler. Germany.

Kelly, J. (2012). Leisure Business Strategies. What They Don't Teach in Business School. Sagamore Publishing LLC.

Montero, M. (Coordinadora). (2007). Dialogando con el constructivismo: Visiones y versiones. Colección DINNOVA. Universidad Autónoma de Ciudad Juárez, México.

Programa de Implementación del Modelo Educativo /PIME/ (2012). Curso de Introducción al Modelo Educativo. Programa de Implementación del Modelo Educativo. Universidad Autónoma de Ciudad Juárez (Inédito).

Orengo, J. (2012). Perspectiva cognoscitiva. Teoría Sociocultural Lev S. Vygotsky. http://www.suagm.edu/umet/biblioteca/Reserva_Profesores/janette_orengo_educ_173/Teori a_\%20Sociocultural_\%20de_\%20Vygotsky_agosto_2012.pdf [recuperado el 20 de julio de 2015].

Sandoval, E. (2006). Guía para realizar prácticas de campo. Universidad Autónoma del Estado de México. http://www.insumisos.com/lecturasinsumisas/GUIA\%20DE\%20CAMPO\%20PARA\%20AN TROPOLOGOS.pdf [recuperado el 28 de agosto de 2015].

Sarasola, J. (2011). Prácticas en trabajo social de la Universidad Pablo de Olavide: 2007-2011. http://www10.ujaen.es/sites/default/files/users/factra/Congreso/56.pdf [recuperado el 2 de septiembre de 2015].

Traverso, D; F. Vanzo; N. Santos; D. Poletto; A. Fischer; J. Feger (2010). Aprendizaje a través de la experiencia y la formación de los gerentes de Agencias de eventos. Un estudio de caso en los municipios de Herval D’Oeste y Joaçaba - SC - Brasil. Estudios y Perspectivas en Turismo, volumen 19 (2010), pp. 359 -381. 
Universidad Autónoma del Estado de México /UAEM/ (2011). Lineamientos para la realización de Prácticas Académicas de Campo de la Universidad Autónoma del Estado de México. Decreto aprobado en sesión ordinaria celebrada el 29 de enero de 2011, por el H. Consejo Universitario de la Universidad Autónoma del Estado de México. http://www.uaemex.mx/fciencias/DesarrolloEstudiantil/MarcoLegal/Lineamientos_para_Rea lizacion_Practicas_Campo.pdf [recuperado el 2 de septiembre de 2015].

Universidad Nacional Autónoma de México /UNAM/ (2012). Lineamientos Generales para la Realización de las Prácticas de Campo de la UNAM. Publicado en Gaceta UNAM el 13 de agosto de 2012. https://consejo.unam.mx/comisiones/especial-de-seguridad/reglamentos-ylineamientos/109-pr\%C3\%A1cticas-de-campo [recuperado el 2 de septiembre de 2015]. 